\title{
Contact-Free Mapping of Electronic Transport Phenomena of Polar Domains in $\mathrm{SrMnO}_{3}$ Films
}

\author{
J. Schaab, ${ }^{1}$ I. P. Krug, ${ }^{2}$ H. Doğanay, ${ }^{3}$ J. Hackl, ${ }^{3}$ D. M. Gottlob, ${ }^{3}$ M. I. Khan, ${ }^{3}$ S. Nemšák, ${ }^{3}$ L. Maurel,${ }^{4}$ \\ E. Langenberg, ${ }^{5}$ P. A. Algarabel, ${ }^{5}$ J. A. Pardo, ${ }^{4,7}$ C. M. Schneider, ${ }^{3}$ and D. Meier ${ }^{1,6, *}$ \\ ${ }^{1}$ Department of Materials, ETH Zürich, 8093 Zürich, Switzerland \\ ${ }^{2}$ Institut für Optik und Atomare Physik, TU Berlin, 10623 Berlin, Germany \\ ${ }^{3}$ Peter Grünberg Institute (PGI-6), Forschungszentrum Jülich, 52425 Jülich, Germany \\ ${ }^{4}$ Instituto de Nanociencia de Aragón, Universidad de Zaragoza, Mariano Esquillor, \\ 50018 Zaragoza, Spain \\ ${ }^{5}$ Instituto de Ciencia de Materiales de Aragón, Universidad de Zaragoza-CSIC, \\ Pedro Cerbuna 12, 50009 Zaragoza, Spain \\ ${ }^{6}$ Department of Materials Science and Engineering, Norwegian University of Science and Technology, \\ 7043 Trondheim, Norway \\ ${ }^{7}$ Departamento de Ciencia y Tecnología de Materiales y Fluidos, Universidad de Zaragoza, \\ 50018 Zaragoza, Spain
}

(Received 8 December 2015; revised manuscript received 24 March 2016; published 13 May 2016)

\begin{abstract}
High-resolution mapping of electronic transport phenomena plays an increasingly important role for the characterization of ferroic domains and their functionality. At present, spatially resolved electronic transport data are commonly gained from local two-point measurements, collected in line-by-line scans with a conducting nanosized probe. Here, we introduce an innovative experimental approach based on lowenergy electron microscopy. As a model case, we study polar domains of varying conductance in strained $\mathrm{SrMnO}_{3}$. By a direct comparison with conductive atomic force and electrostatic force microscopy, we reveal that the applied low-energy electron-microscopy experiment can be considered as an inverse $I(V)$ measurement, providing access to the local electronic conductance with nanoscale resolution and short data-acquisition times in the order of $10-10^{2} \mathrm{~ms}$. Low-energy electrons thus hold yet unexplored application opportunities as a minimal-invasive probe for local electronic transport phenomena, opening a promising route towards spatially resolved, high-throughput sampling at the nanoscale.
\end{abstract}

DOI: 10.1103/PhysRevApplied.5.054009

\section{INTRODUCTION}

Domains in ferroic materials exhibit characteristic transport properties that can be controlled by switching the associated order parameter or, more locally, by inducing minute domain-wall movements. Because of the manipulable transport properties, such domains offer great application potential enabling, for instance, reversible control of local rectification currents [1] or nondestructive resistive readout of memory devices [2,3]. Additional functionality arises when the domain walls display electronic properties that are different from the surrounding domains [4], including anomalous photovoltaic effects in the presence of conducting walls [5] and the formation of chargeable nanocapacitors separated by insulating walls [6]. To date, such local electronic transport properties are commonly analyzed by scanning probe microscopy using either conductive atomic force microscopy (cAFM), i.e., two-point conductance measurements, or electrostatic force microscopy (EFM), which allows for contact-free mapping of spatial conductance variations [7]. Because of

*dennis.meier@ntnu.no the applied line-by-line scanning, however, conductance mapping by cAFM and EFM is time consuming with data-acquisition times in the order of minutes. In addition, the spatial resolution is ultimately limited by the diameter of the probe tip ( $\gtrsim 30 \mathrm{~nm}$ ) [8,9]. A promising but largely unexplored pathway for improving these odds is the application of electron microscopy. The sensitivity to local transport phenomena of scanning electron microscopy [10-12] and x-ray photoemission electron microscopy [13] has already been shown and first attempts have been made to use these techniques for spatially resolved conductance measurements. A conclusive relation to the local $I(V)$ characteristics, however, has not been verified and due to the irradiation of highly energetic electrons in scanning electron microscopy and photons in $\mathrm{x}$-ray photoemission electron microscopy, unwanted irreversible changes in the electronic surface structure may occur. Thus, fast and noninvasive mapping of electronic transport phenomena at the nanoscale is yet to be achieved, gaining even more importance with respect to the future need for adequate monitoring of fabrication processes of envisioned domain- and domain-wall-based nanoelectronics devices. 
Here, we demonstrate contact-free nanoscale characterization of electronic transport properties by performing low-energy electron microscopy (LEEM) at variable electron-gun currents. As a model case we investigate strained $\mathrm{SrMnO}_{3}$ because the system exhibits a unique pattern of polar nanodomains of varying electronic conductance but otherwise uniform structural and electrostatic surface properties. This domain configuration allows for studying transport phenomena without interfering contrasts from unknown topography effects or stationary charges. Based on complementary cAFM and EFM scans we develop a mathematical framework and show that the LEEM experiments on $\mathrm{SrMnO}_{3}$ can be considered as an inverse $I(V)$ measurement: Instead of applying a voltage to the sample, a current $I$ is injected by the electron gun so that a negative voltage $V$ builds up. Since current injection and voltage detection are both realized by the LEEM electron beam, no additional electrical contacts are required. Thus, the current-induced voltage can readily be used for a fast derivation of conductance maps with exposure times of a few milliseconds. Analogous to CAFM, our technique probes the electronic conductance normal to the sample surface, while additional in-plane variations become visible due to the lateral resolution. Our results provide novel insight to the electronic conductance of the new functional material $\mathrm{SrMnO}_{3}$ and reveal LEEM as a promising tool for fast and noninvasive derivation of conductance maps with nanoscale precision.

\section{RESULTS AND DISCUSSION}

For our studies, $\mathrm{SrMnO}_{3}$ epitaxial thin films of $20-\mathrm{nm}$ thickness are grown by pulsed laser deposition on (001)oriented $\left(\mathrm{LaAlO}_{3}\right)_{0.3}\left(\mathrm{Sr}_{2} \mathrm{AlTaO}_{6}\right)_{0.7}$ (LSAT) substrates with $1.7 \%$ tensile strain. Below $T_{C}=400 \mathrm{~K}$ the strained films develop a distinct pattern of four domain states with an in-plane polar axis pointing along the $\langle 110\rangle$ directions of the tetragonal unit cell as detailed elsewhere [6]. Most importantly for our study, $\mathrm{SrMnO}_{3}$ displays well-defined nanodomains of varying conductance at room temperature. Electronic conductance of the domains has been presented in Ref. [6] by cAFM and EFM, but without clarifying the underlying conduction mechanism. To date, the electronic conductance has been addressed only at the bulk level in $\mathrm{SrMnO}_{3}$ polycrystals and superlattices [14,15] so that additional information about local phenomena and emergent contact resistance in cAFM measurements is highly desirable.

\section{A. Electronic transport probed by scanning probe microscopy}

We begin our analysis by considering the mechanism responsible for the electronic conductance in our strained $\mathrm{SrMnO}_{3}$ films. Figure 1(a) displays the spatially resolved current distribution measured by $\mathrm{CAFM}$, recorded on a commercial atomic force microscope (NT-MDT NTEGRA) using Pt-coated silicon tips. Different domains of varying conductance are clearly distinguishable (bright corresponds (a)

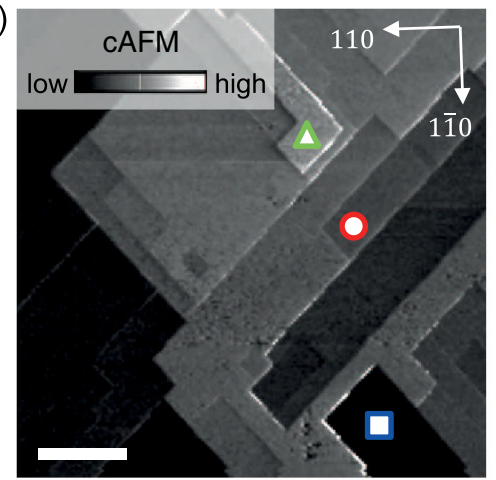

(c)

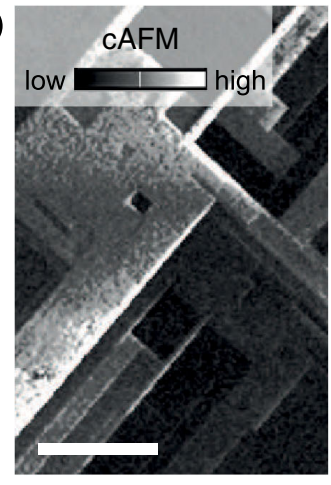

(d) (b)
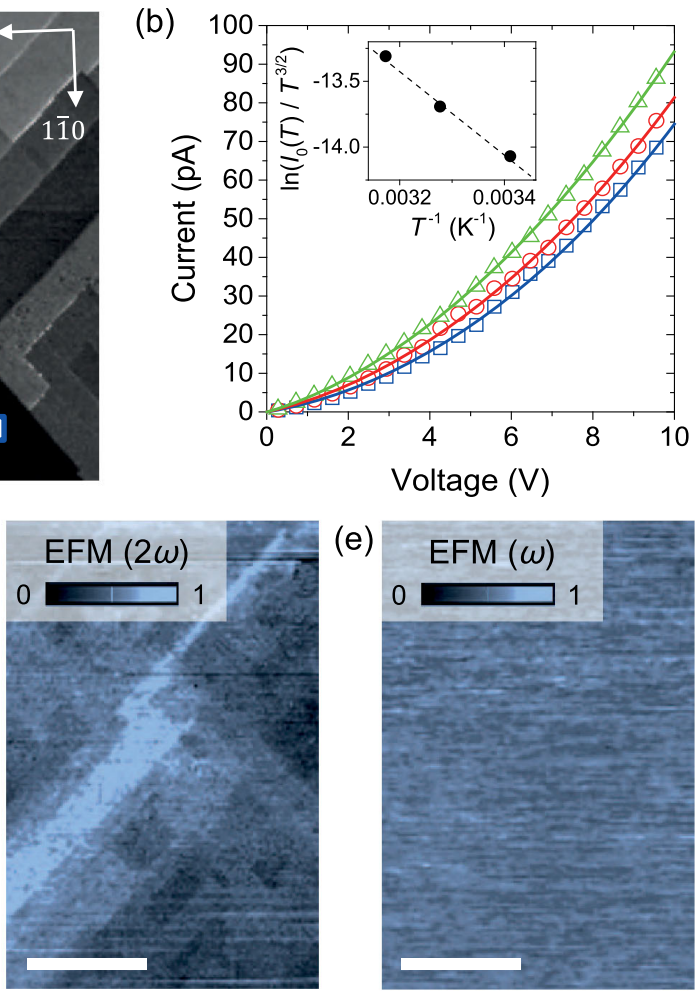

(e)

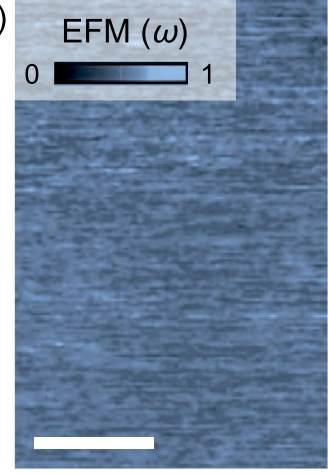

FIG. 1. Transport properties of strained $\mathrm{SrMnO}_{3}$ probed by scanning probe microscopy. (a) cAFM scan obtained with a sample bias of $0.2 \mathrm{~V}$, revealing nanodomains of varying conductance. Scale bar, $2 \mu \mathrm{m}$. (b) Local $I(V)$ curves recorded at the spots marked in (a). Solid lines represent fits to the $I(V)$ curves according to Eq. (2). The inset shows an Arrhenius plot of the temperaturedependent fit constant $I_{0}(T)$ of Eq. (2) from measurements on a single domain. (c) cAFM image recorded at a different sample position. Scale bar, $5 \mu \mathrm{m}$. (d) Normalized $\operatorname{EFM}(2 \omega)$ image obtained at the same sample position shown in (c). The EFM scan reveals the spatial distribution of mobile charge carriers, imaged in the noncontact mode. Scale bar, $5 \mu \mathrm{m}$. (e) Corresponding $\operatorname{EFM}(\omega)$ image recorded at the same position as the scans in (c) and (d) reflecting a homogeneous electrostatic surface potential with no indication of domain-related stationary charges. Scale bar, $5 \mu \mathrm{m}$. 
to high current values), reflecting locally structured conductivity that changes abruptly from one domain to the next. Corresponding $I(V)$ data, evaluated for three different domains, are presented in Fig. 1(b) and their nonlinearity indicates non-Ohmic behavior. The findings presented in Figs. 1(a) and 1(b) are in qualitative agreement with the literature [6], with the difference being that the current values obtained in this work are about 2 orders of magnitude larger. The latter is indicative of a higher oxygen deficiency, but has no detectable effect on the characteristics of the domain pattern [see Fig. 1(a)].

In order to describe the observed non-Ohmic $I(V)$ characteristics in Fig. 1(b), we compare possible transport mechanisms, including thermionic [16-18] and PooleFrenkel emission $[19,20]$, space-charge-limited conduction [21,22], as well as Fowler-Nordheim tunneling [23]. Best fits are achieved assuming the modified thermionic emission model by Simmons [17], which relates the current $I$ to the electric field $\mathcal{E}$ according to

$$
I(\mathcal{E})=A_{\text {eff }} \alpha T^{3 / 2}\left(m^{*} / m_{0}\right)^{3 / 2} \mu \exp \left(-\frac{\phi_{B}}{k T}\right) \mathcal{E} \exp (\beta \sqrt{\mathcal{E}}) .
$$

Here, $A_{\text {eff }}$ is the effective contact area, $\phi_{B}$ is the potential barrier at the metal-insulator (tip-surface) interface, $\mu$ is the electron mobility within the bulk, and $m^{*} / m_{0}$ is the ratio between the effective and the free mass of the electron $\left[\alpha=7.74 \times 10^{-4} \mathrm{As} /\left(\mathrm{cm}^{3} \mathrm{~K}^{3 / 2}\right)\right.$, $\left.\beta=(e / k T) \sqrt{e /\left(4 \pi \epsilon_{0} \epsilon\right)}\right]$. The electric field under the AFM probe tip can be written as $\mathcal{E}(V)=V f(V, \xi)$ with $f(V, \xi)$ accounting for nonlinearities in the voltage (e.g., built-in potentials) and the electric-field distribution $\xi$ $[9,18,24]$. By defining $I_{0}(T)=I_{00} T^{3 / 2} \exp \left[-\left(\phi_{B} / k T\right)\right]$ and $V_{0}^{-1}=\beta^{2} f(V, \xi)$, Eq. (1) then becomes

$$
I(V)=I_{0}(T) \frac{V}{V_{0}} \exp \left(\sqrt{\frac{V}{V_{0}}}\right) .
$$

Corresponding fits (solid lines) are presented together with the measured $I(V)$ data in Fig. 1(b). The agreement between model and experiment holds for all measured temperatures between room temperature and $315 \mathrm{~K}$ (not shown). From the temperature-dependent data an average potential barrier $\phi_{B}$ can be derived by plotting $\ln \left[I_{0}(T) / T^{3 / 2}\right]$ as a function of $1 / T$ [see the inset to Fig. 1(b)]. The data yield $\phi_{B}=0.27 \pm 0.03 \mathrm{eV}$ and no indication of pronounced domain-dependent variations. Based on $\phi_{B}$ and the fits in Fig. 1(b) a rough estimate of the average electron mobility $\mu$ at room temperature can be achieved when assuming a circular tip-surface contact $\left(A_{\text {eff }}=\pi r_{\text {tip }}^{2}, r_{\text {tip }}=30 \mathrm{~nm}\right)$. In addition, an upper limit for the dielectric constant $\epsilon$ can be derived assuming a constant electric field over the film $\left[f(V, \xi)=1 / d_{\text {film }}\right.$, $\left.d_{\text {film }}=20 \mathrm{~nm}\right]$. The latter corresponds to a rather drastic but common simplification of the actual electric-field distribution in cAFM $[20,25,26]$. We note that in our specific case, where the $\mathrm{SrMnO}_{3}$ film thickness is smaller than the radius of the probe tip, this simplification yields an upper limit for $\mathcal{E}\left(\mathcal{E} \approx V / d_{\text {film }}>V / r_{\text {tip }}\right)$, leading to $\mu \approx(1.4 \pm 0.3) \times 10^{-3} \mathrm{~cm}^{2} /(\mathrm{V} \mathrm{s})$ (for $m^{*}=m_{0}$ ) and $\epsilon \leq 490 \pm 130$. The estimated electron mobility is comparable to perovskite oxides such as $\mathrm{CaMnO}_{3-\delta}$ and $\mathrm{Ba}_{0.5} \mathrm{Sr}_{0.5} \mathrm{TiO}_{3}$ where $\mu$ is in the order of $10^{-2} \mathrm{~cm}^{2} /(\mathrm{Vs})$ and $10^{-3} \mathrm{~cm}^{2} /(\mathrm{V} \mathrm{s})$, respectively $[27,28]$. The derived upper limit for the dielectric constant $\epsilon$ is consistent with frequency-dependent results obtained at low temperature $[\epsilon(1 \mathrm{MHz})=110$ at $T=5 \mathrm{~K}]$ [29]. Based on the excellent agreement between model and data we thus conclude that the electronic conductance in strained $\mathrm{SrMnO}_{3}$ is dominated by the interface-controlled and bulk-limited [24,30] transport mechanism described by Eqs. (1) and (2). This implies that the mean free path of the charge carriers in $\mathrm{SrMnO}_{3}$ is smaller than the thickness of the measured film, i.e., shorter than $20 \mathrm{~nm}$. As a consequence, both the Schottky barrier formed at the tip-surface interface and the mobility of the carriers in the bulk determine the transport behavior [17]. The charge carriers are affected by traps and interface states, which gives rise to additional scattering within the bulk compared to standard Schottky emission [31].

In order to obtain direct evidence for the impact of bulk contributions on the electronic conductance and collect additional information about local variations of the barrier $\phi_{B}$, we compare cAFM with complementary EFM measurements as presented in Figs. 1(c)-1(e). The EFM maps of mobile [Fig. 1(d)] and fixed [Fig. 1(e)] charges are obtained simultaneously by recording the EFM signals at $2 \omega$ and $\omega$, respectively, while scanning in noncontact mode with an ac voltage applied to the tip $(\omega=41.3 \mathrm{kHz}$, $\left.U_{p p}=14 \mathrm{~V}\right)[32,33]$. The $\operatorname{EFM}(2 \omega)$ image in Fig. 1(d) shows the same pattern as probed by cAFM [Fig. 1(c)], which proves that the intrinsic bulk properties play a significant role for the domain conductance. The simultaneously recorded $\operatorname{EFM}(\omega)$ image in Fig. 1(e) reveals a homogeneous electrostatic surface potential. The latter is in agreement with the in-plane orientation of the polar axis in $\mathrm{SrMnO}_{3}$ and excludes the presence of domain-specific stationary surface charges that may locally alter $\phi_{B}$. The EFM data thus corroborate the proposed interface-controlled, bulklimited transport mechanism and the material properties derived based on the cAFM experiments.

\section{B. Current-induced potentials visualized by low-energy electron microscopy}

After elaborating the electronic transport mechanism in the strained $\mathrm{SrMnO}_{3}$ film in terms of conventional scanning probe microscopy methods, we next discuss the potential of LEEM for deriving conductance maps. LEEM is a 
(a)

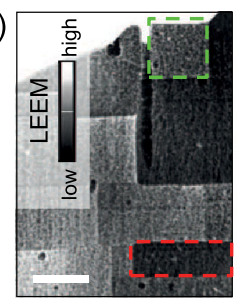

(b)

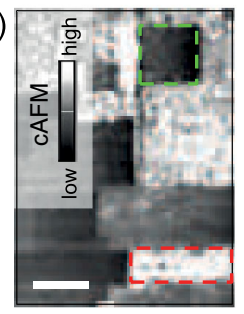

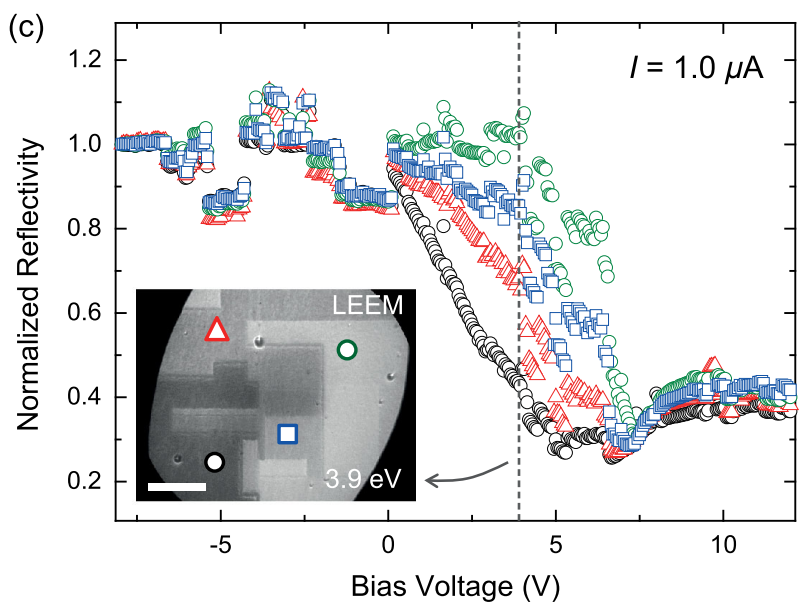

FIG. 2. LEEM reflectivity measurements. (a) and (b) LEEM and cAFM images recorded at the same position. Green and red dashed lines highlight examples for insulating and conductive domains, respectively, which lead to opposite contrasts in LEEM compared to cAFM. The LEEM image in (a) is obtained with an exposure time of $500 \mathrm{~ms}$. A closer inspection of (a) and (b) further reveals differences in the relative contrasts. This is because cAFM resolves variations in current $\Delta I\left(V_{\text {const }}\right)$, while LEEM measures differences in the surface potential $\Delta V\left(I_{\text {const }}\right)$, which are different due to the nonlinear $I(V)$ characteristic of $\mathrm{SrMnO}_{3}$ [see Fig. 1(b)]. Scale bars, $2 \mu \mathrm{m}$. (c) MEM-LEEM transition measured with an electron-gun current of $1.0 \mu \mathrm{A}$. Different curves correspond to the different domains marked in the inset and, for comparison, are normalized with respect to the reflectivity obtained below $-7 \mathrm{eV}$. The inset shows a LEEM image taken with an exposure time of $250 \mathrm{~ms}$ at a bias voltage of $3.9 \mathrm{eV}$, indicated by the dashed line in (c). Scale bar, $2 \mu \mathrm{m}$.

well-established and explicitly powerful method for characterizing, e.g., the surface structure of metals and semiconductors in real time and with nanoscale resolution ( $\gtrsim 2 \mathrm{~nm}$ ) [34]. More recently, LEEM was applied for the analysis and imaging of domains in ferroic oxides [35-37], but the sensitivity of LEEM towards relative, local conductance variations $[38,39]$ has not been explored in detail. In the maybe closest approach, Kautz and co-workers studied the conductivity of in-plane biased graphene based on potentiometry measurements [40]. Their method is particularly strong for measuring variations in the conductance parallel to the surface in electrically conducting materials. In order to expand their approach towards poorly conducting or insulating materials, however, either high in-plane voltages or smaller electrode distances are required, both interfering with the imaging by low-energy electrons. Complementary LEEM measurements probing the out-of-plane conductance, that is normal to the sample surface, are virtually nonexisting. We thus begin with a direct comparison of the spatially resolved LEEM and CAFM data presented in Figs. 2(a) and 2(b). The LEEM data are recorded under ultrahigh vacuum $\left(6 \times 10^{-10} \mathrm{mbar}\right)$ at the UE56-1 SGM beam line of the Forschungszentrum Jülich, BESSY-II storage ring, Helmholtz-Zentrum Berlin using a novel type of aberration-corrected electron microscope (SPECS FE-LEEM P90 AC).

Figures 2(a) and 2(b) are taken at the same position and demonstrate that standard reflectivity measurements resolve a domain pattern that qualitatively matches with the cAFM conductance map. The LEEM contrast levels, however, are inverted with respect to cAFM, i.e., in the
LEEM image conducting domains are darker than insulating domains. This behavior is consistent with bandstructure-based models relating higher (lower) reflectivity in LEEM to a smaller (larger) number of empty states at the respective kinetic energy [34]. In order to gain more detailed insight, we perform reflectivity measurements with varying kinetic energy of the electrons. The latter is achieved by applying a variable bias voltage to the sample. The results obtained for bias voltages between -8 and $+12 \mathrm{~V}$ at a constant electron-gun current $I=1 \mu \mathrm{A}$ are summarized in Fig. 2(c). Figure 2(c) shows the average normalized electron reflectivity evaluated for the four domains marked in the LEEM image in the inset. For all domains a pronounced steplike drop in electron reflectivity is obtained for increasing bias voltage, indicating the so-called MEM-LEEM transition; that is the transition from the mirror-electron-microscopy (MEM) regime of high reflectivity to the energy range where electrons start impinging onto the surface. Since the nature of the energydependent change in reflectivity is nontrivial, the voltage $\tilde{V}$ at which the MEM-LEEM transition occurs is often defined based on the point of inflection between maximum and minimum reflectivity [36,41,42].

For the domains highlighted in the inset to Fig. 2(c) the characteristic voltage $\tilde{V}$ shifts by up to $\approx 5 \mathrm{~V}$. Such local shifts in $\tilde{V}$ lead to the strong domain contrasts in LEEM images obtained in the transition region. Typical sources for such shifts in the MEM-LEEM transition are spatial variations in surface topography, surface potential, or surface polarization, with the latter determining the internal and external screening, band bending, and electron affinity 
at the surface $[34,35,37]$. Contributions from the aforementioned sources, however, can be excluded based on our scanning-probe-microscopy data, which reveal a homogeneous surface topography with a root mean square roughness of only $0.4 \pm 0.1 \mathrm{~nm}$ (not shown) and no electrostatic contributions as presented in Fig. 1(e). These findings, in addition to the close relation between the LEEM reflectivity data [Fig. 2(a)] and cAFM conductance map [Fig. 2(b)], unambiguously demonstrate that the LEEM contrasts obtained on $\mathrm{SrMnO}_{3}$ originate from potential differences that relate to variations in conductance $G$.

\section{Conductance maps gained by low-energy electron microscopy}

In order to strengthen our conclusion and elaborate the relation between emergent LEEM contrasts and the local conductance we devised an innovative strategy, performing LEEM experiments as a function of the electron-gun current (see Fig. 3). Taking into account that the inelastic mean free path of low-energy electrons in inorganic compounds can reach several nanometers [43], the experiment may be considered as an inverse $I(V)$ measurement complementary to the cAFM data in Fig. 1(b): Instead of applying a voltage to the sample, a current $I$ is injected by the electron gun and a negative voltage is built up at the surface of the sample due to the finite electronic conductance of $\mathrm{SrMnO}_{3}$. In the LEEM data in Fig. 3(a) the effect is evident from the trend that the MEM-LEEM transition voltage $\tilde{V}$ continuously shifts towards higher values with increasing gun current. As we show in Fig. 3(a) this trend, i.e., the current-induced change in voltage, is captured by the transport model of Simmons [Eq. (2)], which enables a quantitative analysis. Since it is the electron-gun current that induces the voltage, however, it is reasonable to use the inverse of Eq. (2) for analyzing the dose-dependent LEEM experiment

$$
V(I)=4 V_{0} \times W^{2}\left(\frac{1}{2} \sqrt{\frac{I}{I_{0}(T)}}\right),
$$

where $W$ denotes the so-called Lambert- $W$ function $\left[z=W(z) e^{W(z)}, \quad z \in \mathbb{C}\right]$. Analogous to the transport measurements in terms of cAFM (see Sec. II A), the electric-field distribution and potential barrier are the only experimental input parameters that require additional assumptions. Obviously, these two parameters are not identical for CAFM and LEEM. Nevertheless, when rigorously applying the same approximation, i.e., $V(I)=\tilde{V}$, $m^{*}=m_{0}$, and an average barrier $\phi_{B}=0.27 \mathrm{eV}$, we find values for the electron mobility $[\mu=(1.1 \pm 1.0) \times$ $\left.10^{-3} \mathrm{~cm}^{2} /(\mathrm{V} \mathrm{s})\right]$ and dielectric constant $(\epsilon=80 \pm 8)$ that are in reasonable agreement with our transport results (area illuminated by the electron gun: $A_{\text {eff }}=77 \pm 3 \mu \mathrm{m}^{2}$ ). The agreement between the material constants derived based on cAFM and LEEM justifies the approximation applied for fitting Eq. (3) to the LEEM data. Most importantly, the fact that both the cAFM and LEEM results can be described consistently based on the same transport model further strengthens the conclusion that variations in $\tilde{V}$ are a direct measure for changes in conductance $(G=I / \tilde{V})$.

This correlation allows for generating conductance maps by LEEM as presented in Figs. 3(b) and 3(c). The conductance maps for 0.1 and $2.0 \mu \mathrm{A}$ in Figs. 3(b) and 3(c) represent the local conductance normal to the (a)

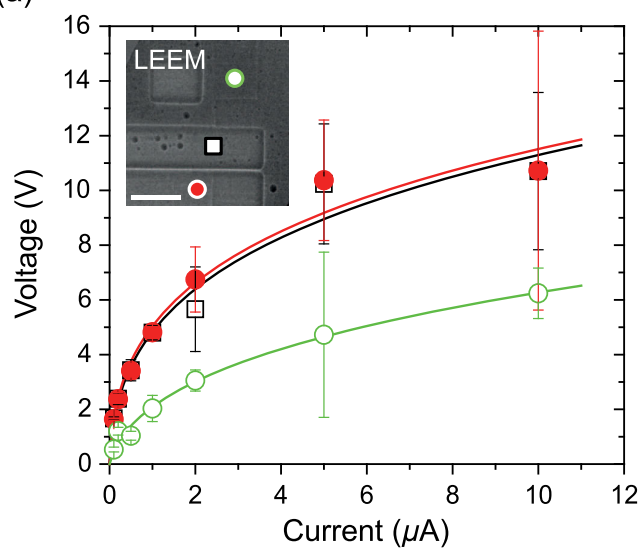

(b)

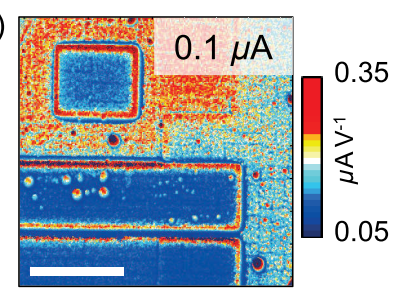

(c)

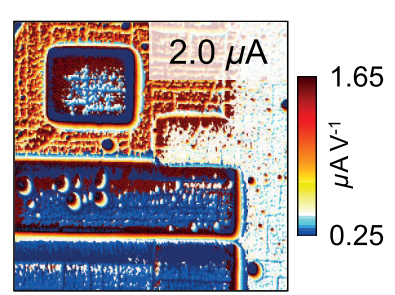

FIG. 3. Current-induced voltage and conductance maps derived by LEEM. (a) Data points present the detected MEM-LEEM transition voltage $\tilde{V}$ as a function of the gun current $I$ for the three domains marked in the LEEM image in the inset. The LEEM image is obtained at $1.0 \mathrm{~V}$ bias voltage with $I=1.0 \mu \mathrm{A}$. Scale bar, $2 \mu \mathrm{m}$. Each data point is obtained from a series of reflectivity measurements analogous to the data presented in Fig. 2(c). For increasing gun currents the MEM-LEEM transition voltage shifts to higher energies, which is captured by fitting the $V(I)$ characteristics described by Eq. (3) to the LEEM data with $V(0)=0$ (solid lines). (b) and (c) Conductance maps obtained for gun currents of 0.1 and $2.0 \mu \mathrm{A}$ at the position shown in the inset to (a). Colors represent the local conductance $G$. Scale bar in (b), $2 \mu \mathrm{m}$. 
sample surface and are generated based on $\tilde{V}$ values extracted pixel by pixel from reflectivity measurements, as seen in Fig. 2(c). Figures 3(b) and 3(c) reflect that the sensitivity of conductance maps increases with increasing electron-gun currents, which is consistent with Fig. 3(a). The gain in sensitivity, however, coincides with a loss in spatial resolution because higher gun currents yield more pronounced charging effects that reduce the image quality. In general, the conductance resolution can be optimized up to the point at which built-up charges prevent arriving electrons from interacting with the sample and hence cancel the imaging procedure. For the model system $\mathrm{SrMnO}_{3}$ an electron-gun current of $0.1 \mu \mathrm{A}$, for instance, allows for resolving conductance variations $\Delta G \approx 30 \mathrm{nA} / \mathrm{V}$ with a spatial resolution $\lesssim 100 \mathrm{~nm}$.

\section{SUMMARY AND CONCLUSION}

In summary, by applying cAFM and EFM we demonstrate interface-controlled and bulk-limited transport in strained $\mathrm{SrMnO}_{3}$ and derive estimates for the potential barrier, electron mobility, and dielectric constant. We then compare the scanning probe results with LEEM measurements and reveal a correlation between the transport behavior and LEEM reflectivity data. This correlation allows for contact-free imaging of current-induced potential variations, i.e., visualizing areas of different conductance based on low-energy electrons with data-acquisition times in the order of a $10-10^{2} \mathrm{~ms}$ [6]. The applied LEEM reflectivity measurements provide qualitative information with a high spatial resolution that is limited only by the performance of the microscope. Quantitative conductance maps are gained based on LEEM experiments performed at variable electron-gun current. Emergent contrasts are dominated by the dc bulk conductance normal to the sample surface with in-plane variations becoming visible due to the lateral resolution. The application of dosedependent LEEM facilitates noninvasive conductance maps with adjustable sensitivity and spatial resolution and, most importantly, provides access to local $I(V)$ characteristics. Our concept relies on surface charging which is a well-known and widespread effect in electron microscopy, occurring in a large variety of functional materials including, e.g., semiconductors, insulators, ferroelectrics, and silicon-based structures. With this, our results foreshadow possible applications in fundamental materials science, such as time-resolved studies of dynamical transport phenomena, but even beyond the basic research sector. The use of electron beams is already common in industry and even waver-sized samples can be characterized automatically and quickly. Moreover, the usage of a single electron beam for both current injection and noninvasive probing can easily be adapted for industrial purposes. Thus, transport measurements by low-energy electrons may be employed for industrial sampling and quality monitoring in nanoelectronics fabrication processes.

\section{ACKNOWLEDGMENTS}

The authors thank Andres Cano for many enlightening discussions, Martin Lilienblum for technical assistance, and Manfred Fiebig for financial and scientific support. We thank HZB for the allocation of synchrotron beam time and we thankfully acknowledge financial support by HZB. Research at the ETH was financed in part by the SNF (Proposal No. 200021_149192). L. M., E. L., P. A. A., and J. A. P. acknowledge financial support from Ministerio de Economía y Competitividad under Project No. MAT201451982-C2 and Gobierno de Aragón under Project No. E26.

[1] Weida Wu, J. R. Guest, Y. Horibe, S. Park, T. Choi, S.-W. Cheong, and M. Bode, Polarization-Modulated Rectification at Ferroelectric Surfaces, Phys. Rev. Lett. 104, 217601 (2010).

[2] V. Garcia, S. Fusil, K. Bouzehouane, S. Enouz-Vedrenne, N. D. Mathur, A. Barthélémy, and M. Bibes, Giant tunnel electroresistance for non-destructive readout of ferroelectric states, Nature (London) 460, 81 (2009).

[3] A. Gruverman, D. Wu, H. Lu, Y. Wang, H. W. Jang, C. M. Folkman, M. Ye. Zhuravlev, D. Felker, M. Rzchowski, C.-B. Eom, and E. Y. Tsymbal, Tunneling electroresistance effect in ferroelectric tunnel junctions at the nanoscale, Nano Lett. 9, 3539 (2009).

[4] Dennis Meier, Functional domain walls in multiferroics, J. Phys. Condens. Matter 27, 463003 (2015).

[5] S. Y. Yang, J. Seidel, S. J. Byrnes, P. Shafer, C.-H. Yang, M. D. Rossell, Y.-H. Chu, J. F. Scott, J. W. Ager, III, L. W. Martin, and R. Ramesh, Above-bandgap voltages from ferroelectric photovoltaic devices, Nat. Nanotechnol. 5, 143 (2010).

[6] Carsten Becher, Laura Maurel, Ulrich Aschauer, Martin Lilienblum, César Magén, Dennis Meier, Eric Langenberg, Morgan Trassin, Javier Blasco, Ingo P. Krug, Pedro A. Algarabel, Nicola A. Spaldin, José A. Pardo, and Manfred Fiebig, Strain-induced coupling of electrical polarization and structural defects in $\mathrm{SrMnO}_{3}$ films, Nat. Nanotechnol. 10, 661 (2015).

[7] Huiseong Jeong, Kyung Moon Lee, Y. H. Ahn, Soonil Lee, and Ji-Yong Park, Non-contact local conductance mapping of individual graphene oxide sheets during the reduction process, J. Phys. Chem. Lett. 6, 2629 (2015).

[8] B. Cappella and G. Dietler, Force-distance curves by atomic force microscopy, Surf. Sci. Rep. 34, 1 (1999).

[9] Amit Kumar, Thomas M. Arruda, Alexander Tselev, Ilia N. Ivanov, Jamie S. Lawton, Thomas A. Zawodzinski, Oleg Butyaev, Sergey Zayats, Stephen Jesse, and Sergei V. Kalinin, Nanometer-scale mapping of irreversible electrochemical nucleation processes on solid Li-ion electrolytes, Sci. Rep. 3, 1621 (2013).

[10] V. V. Aristov, L. S. Kokhanchik, K.-P. Meyer, and H. Blumtritt, Scanning electron microscopic investigations of 
peculiarities of the $\mathrm{BaTiO}_{3}$ ferroelectric domain contrast, Phys. Status Solidi A 78, 229 (1983).

[11] V. V. Aristov, L. S. Kokhanchik, and Yu. I. Voronovskii, Voltage contrast of ferroelectric domains of lithium niobate in SEM, Phys. Status Solidi A 86, 133 (1984).

[12] J. Li, H. X. Yang, H. F. Tian, C. Ma, S. Zhang, Y. G. Zhao, and J. Q. Li, Scanning secondary-electron microscopy on ferroelectric domains and domain walls in $\mathrm{YMnO}_{3}, \mathrm{Appl}$. Phys. Lett. 100, 152903 (2012).

[13] J. Schaab, I. P. Krug, F. Nickel, D. M. Gottlob, H. Doğanay, A. Cano, M. Hentschel, Z. Yan, E. Bourret, C. M. Schneider, R. Ramesh, and D. Meier, Imaging and characterization of conducting ferroelectric domain walls by photoemission electron microscopy, Appl. Phys. Lett. 104, 232904 (2014).

[14] C. Chiorescu, J. L. Cohn, and J. J. Neumeier, Impurity conduction and magnetic polarons in antiferromagnetic oxides, Phys. Rev. B 76, 020404 (2007).

[15] S. J. May, T. S. Santos, and A. Bhattacharya, Onset of metallic behavior in strained $\left(\mathrm{LaNiO}_{3}\right)_{n} /\left(\mathrm{SrMnO}_{3}\right)_{2}$ superlattices, Phys. Rev. B 79, 115127 (2009).

[16] W. Schottky, Halbleitertheorie der Sperrschicht, Naturwissenschaften 26, 843 (1938).

[17] J. G. Simmons, Richardson-Schottky Effect in Solids, Phys. Rev. Lett. 15, 967 (1965).

[18] S. M. Sze and Kwok K. Ng, Physics of Semiconductor Devices (John Wiley \& Sons, Ltd., Hoboken, New Jersey, 2007).

[19] J. Frenkel, On pre-breakdown phenomena in insulators and electronic semi-conductors, Phys. Rev. 54, 647 (1938).

[20] P. Zubko, D. J. Jung, and J. F. Scott, Electrical characterization of $\mathrm{PbZr}_{0.4} \mathrm{Ti}_{0.6} \mathrm{O}_{3}$ capacitors, J. Appl. Phys. 100, 114113 (2006).

[21] A. Rose, Space-charge-limited currents in solids, Phys. Rev. 97, 1538 (1955).

[22] Murray A. Lampert and Peter Mark, Current Injection in Solids (Academic Press, Inc., New York and London, 1970).

[23] R. H. Fowler and L. Nordheim, Electron emission in intense electric fields, Proc. R. Soc. A 119, 173 (1928).

[24] L. Pintilie, I. Vrejoiu, D. Hesse, G. LeRhun, and M. Alexe, Ferroelectric polarization-leakage current relation in high quality epitaxial $\mathrm{Pb}(\mathrm{Zr}, \mathrm{Ti}) \mathrm{O}_{3}$ films, Phys. Rev. B 75, 104103 (2007).

[25] J. Seidel, P. Maksymovych, Y. Batra, A. Katan, S.-Y. Yang, Q. He, A. P. Baddorf, S. V. Kalinin, C.-H. Yang, J.-C. Yang, Y.-H. Chu, E. K. H. Salje, H. Wormeester, M. Salmeron, and R. Ramesh, Domain Wall Conductivity in La-Doped $\mathrm{BiFeO}_{3}$, Phys. Rev. Lett. 105, 197603 (2010).

[26] Jill Guyonnet, Iaroslav Gaponenko, Stefano Gariglio, and Patrycja Paruch, Conduction at domain walls in insulating $\mathrm{Pb}\left(\mathrm{Zr}_{0.2} \mathrm{Ti}_{0.8}\right) \mathrm{O}_{3}$ thin films, Adv. Mater. 23, 5377 (2011).

[27] Ekaterina I. Goldyreva, Ilya A. Leonidov, Mikhail V. Patrakeev, and Victor L. Kozhevnikov, Electron transport in $\mathrm{CaMnO}_{3-\delta}$ at elevated temperatures: A mobility analysis, J. Solid State Electrochem. 17, 1449 (2013).

[28] Sufi Zafar, Robert E. Jones, Bo Jiang, Bruce White, V. Kaushik, and S. Gillespie, The electronic conduction mechanism in barium strontium titanate thin films, Appl. Phys. Lett. 73, 3533 (1998).

[29] H. Sakai, S. Ishiwata, D. Okuyama, A. Nakao, H. Nakao, Y. Murakami, Y. Taguchi, and Y. Tokura, Electron doping in the cubic perovskite $\mathrm{SrMnO}_{3}$ : Isotropic metal versus chainlike ordering of Jahn-Teller polarons, Phys. Rev. B 82, 180409 (2010).

[30] J. F. Scott, There's no place like Ohm: Conduction in oxide thin films, J. Phys. Condens. Matter 26, 142202 (2014).

[31] Fu-Chien Chiu, A review on conduction mechanisms in dielectric films, Adv. Mater. Sci. Eng. 2014, 578168 (2014).

[32] F. Johann, Á. Hoffmann, and E. Soergel, Impact of electrostatic forces in contact-mode scanning force microscopy, Phys. Rev. B 81, 094109 (2010).

[33] Paul Girard, Electrostatic force microscopy: Principles and some applications to semiconductors, Nanotechnology 12, 485 (2001).

[34] Ernst Bauer, Surface Microscopy with Low Energy Electrons (Springer Science+Business Media New York, 2014).

[35] Salia Cherifi, Riccardo Hertel, Stéphane Fusil, Hélène Béa, Karim Bouzehouane, Julie Allibe, Manuel Bibes, and Agnès Barthélémy, Imaging ferroelectric domains in multiferroics using a low-energy electron microscope in the mirror operation mode, Phys. Status Solidi 4, 22 (2010).

[36] J. E. Rault, W. Ren, S. Prosandeev, S. Lisenkov, D. Sando, S. Fusil, M. Bibes, A. Barthélémy, L. Bellaiche, and N. Barrett, Thickness-Dependent Polarization of Strained $\mathrm{BiFeO}_{3}$ Films with Constant Tetragonality, Phys. Rev. Lett. 109, 267601 (2012).

[37] N. Barrett, J. E. Rault, J. L. Wang, C. Mathieu, A. Locatelli, T. O. Mentes, M. A. Niño, S. Fusil, M. Bibes, A. Barthélémy, D. Sando, W. Ren, S. Prosandeev, L. Bellaiche, B. Vilquin, A. Petraru, I. P. Krug, and C. M. Schneider, Full field electron spectromicroscopy applied to ferroelectric materials, J. Appl. Phys. 113, 187217 (2013).

[38] Ludwig Mayer, On electron mirror microscopy, J. Appl. Phys. 26, 1228 (1955).

[39] K. N. Maffitt, Interpretation of electron-mirror micrographs of ferroelectric and dielectric surfaces, J. Appl. Phys. 39, 3878 (1968).

[40] J. Kautz, J. Jobst, C. Sorger, R. M. Tromp, H. B. Weber, and S. J. van der Molen, Low-energy electron potentiometry: Contactless imaging of charge transport on the nanoscale, Sci. Rep. 5, 13604 (2015).

[41] R. Le Bihan, J. L. Chartier, and L. Jean, Measurement of surface potential between ferroelectric domains with a quantitative electron mirror microscope, Ferroelectrics 17, 429 (1977).

[42] I. Krug, N. Barrett, A. Petraru, A. Locatelli, T. O. Mentes, M. A. Nio, K. Rahmanizadeh, G. Bihlmayer, and C. M. Schneider, Extrinsic screening of ferroelectric domains in $\mathrm{Pb}\left(\mathrm{Zr}_{0.48} \mathrm{Ti}_{0.52}\right) \mathrm{O}_{3}$, Appl. Phys. Lett. 97, 222903 (2010).

[43] S. Tanuma, C. J. Powell, and D. R. Penn, Calculations of electron inelastic mean free paths. III. Data for 15 inorganic compounds over the 50-2000 eV range, Surf. Interface Anal. 17, 927 (1991). 